\title{
Z+jet production at NNLO
}

\section{A. Gehrmann-De Ridder}

Institute for Theoretical Physics, ETH, CH-8093 Zürich, Switzerland

Department of Physics, University of Zürich, CH-8057 Zürich, Switzerland

Kavli Institute for Theoretical Physics, UC Santa Barbara, Santa Barbara, USA

E-mail: gehra@phys.ethz.ch

\section{T. Gehrmann}

Department of Physics, University of Zürich, CH-8057 Zürich, Switzerland

Kavli Institute for Theoretical Physics, UC Santa Barbara, Santa Barbara, USA

E-mail: thomas.gehrmannduzh.ch

\section{E.W.N. Glover}

Institute for Particle Physics Phenomenology, Department of Physics, University of Durham, Durham, DH1 3LE, UK

E-mail: e.w.n.glover@durham.ac.uk

\section{A. Huss*}

Institute for Theoretical Physics, ETH, CH-8093 Zürich, Switzerland

E-mail: ahussephys.ethz.ch

\section{T.A. Morgan}

Institute for Particle Physics Phenomenology, Department of Physics, University of Durham, Durham, DH1 3LE, UK

E-mail: t.a.morgan@durham.ac.uk

We present our calculation of the next-to-next-to-leading order (NNLO) QCD corrections to Z+ jet production in hadronic collisions. Phenomenological results are presented which comprise various differential distributions for $8 \mathrm{TeV}$ proton-proton collisions. A significant reduction of the scale uncertainties is observed throughout as we move from NLO to NNLO. We further discuss how this calculation can be used to describe the inclusive Z-boson production at large transverse momentum. To this end, the theory prediction is compared to the measurements performed by the ATLAS and CMS collaborations at a centre-of-mass energy of $8 \mathrm{TeV}$. Here, the inclusion of NNLO QCD effects are found to result in a substantial improvement in the agreement between theory and data for the normalised distributions.

38th International Conference on High Energy Physics

3-10 August 2016

Chicago, USA

\footnotetext{
${ }^{*}$ Speaker.
} 


\section{Introduction}

The Drell-Yan-like production of charged lepton pairs, $\mathrm{pp} \rightarrow \mathrm{Z} / \gamma^{*} \rightarrow \ell^{+} \ell^{-}$, is among the most important so-called "standard candle" processes at the LHC, owing to its large production rate and clean experimental signature. It provides the opportunity to test the Standard Model prediction over a large kinematic range and delivers crucial constraints in the fit of parton distribution functions (PDF's). Moreover, it represents a powerful tool for detector calibration and also constitutes an important background to many searches for physics beyond the Standard Model.

The production of a $\mathrm{Z}$ boson in association with a hadronic jet still retains a large event rate and also has the advantage of introducing a direct sensitivity to the strong coupling $\alpha_{\mathrm{s}}$ and the gluon PDF. As such, $\mathrm{Z}+$ jet production provides an ideal testing ground for our understanding of both strong and electroweak interactions in a hadron-collider environment [1,2].

On the theory side, a variety of corrections have been considered in order to improve the accuracy of the theoretical predictions for the Z+jet process. Both the NLO QCD [3] and EW [4] corrections to this process have been known for some time. The NLO multi-jet merging procedure has been recently extended to incorporate both NLO QCD and EW corrections into the MEPs@ NLO framework [5]. QCD corrections to Z+jet production at NNLO were computed in Refs. [6, 7] using two independent methods and have subsequently been validated against each other.

\section{Z-boson production in association with a hadronic jet}

Our calculation of the NNLO corrections to $\mathrm{Z}+$ jet production [6] has been performed using the antenna-subtraction formalism [9]. All results are implemented in a flexible parton-level Monte Carlo program NNLOJET which allows the computation of cross sections and any (multi-) differential distribution with arbitrary (infrared-safe) event-selection cuts at NNLO.

Figure 1 shows the numerical results for Z-boson observables, which comprise the transverse momentum and the rapidity of the di-lepton system. The top frames (a) of the plots display the absolute distributions for the different perturbative orders, while the the relative impact of the (N)NLO corrections are exposed in the bottom panels (b) in terms of the ratio $K=$ $\mathrm{d} \sigma^{(\mathrm{N}) \mathrm{NLO}}(\mu) / \mathrm{d} \sigma^{(\mathrm{N}) \mathrm{LO}}\left(\mu=M_{\mathrm{Z}}\right)$. The theoretical uncertainties shown as bands around the curves are estimated by varying the unphysical scales $\mu \equiv \mu_{\mathrm{R}}=\mu_{\mathrm{F}}$ by the factors $[1 / 2,2]$ from the central scale choice $\mu=M_{\mathrm{Z}}$. For more results and details of the numerical setup, we refer the reader to Refs. $[6,8]$.

The left plot in Fig. 1 shows the transverse momentum distribution of the $\mathrm{Z}$ boson. An interesting structure is observed at around $p_{\mathrm{T}}^{\mathrm{Z}} \approx 30 \mathrm{GeV}$, which can be attributed to the well-known Sudakov shoulder phenomenon [10]. It is caused by the indirect constraint on the leading-order process, $p_{\mathrm{T}}^{\mathrm{Z}}>30 \mathrm{GeV}$, which is alleviated by real-radiation corrections at higher orders. At larger transverse momenta, the NLO corrections are at the level of 30-70\% with a residual scale uncertainty of $5-10 \%$. This uncertainty is significantly reduced as we move from NLO to NNLO and we further observe a stabilisation of the perturbative series with NNLO corrections that increase the prediction by approximately $1 \%$. The rapidity of the $\mathrm{Z}$ boson is shown in the right plot of Fig. 1. The NLO and NNLO corrections are the largest in the forward/backward region where they can reach corrections of up to $\sim 90 \%$ and $\sim 20 \%$, respectively. In the central region, the NNLO 

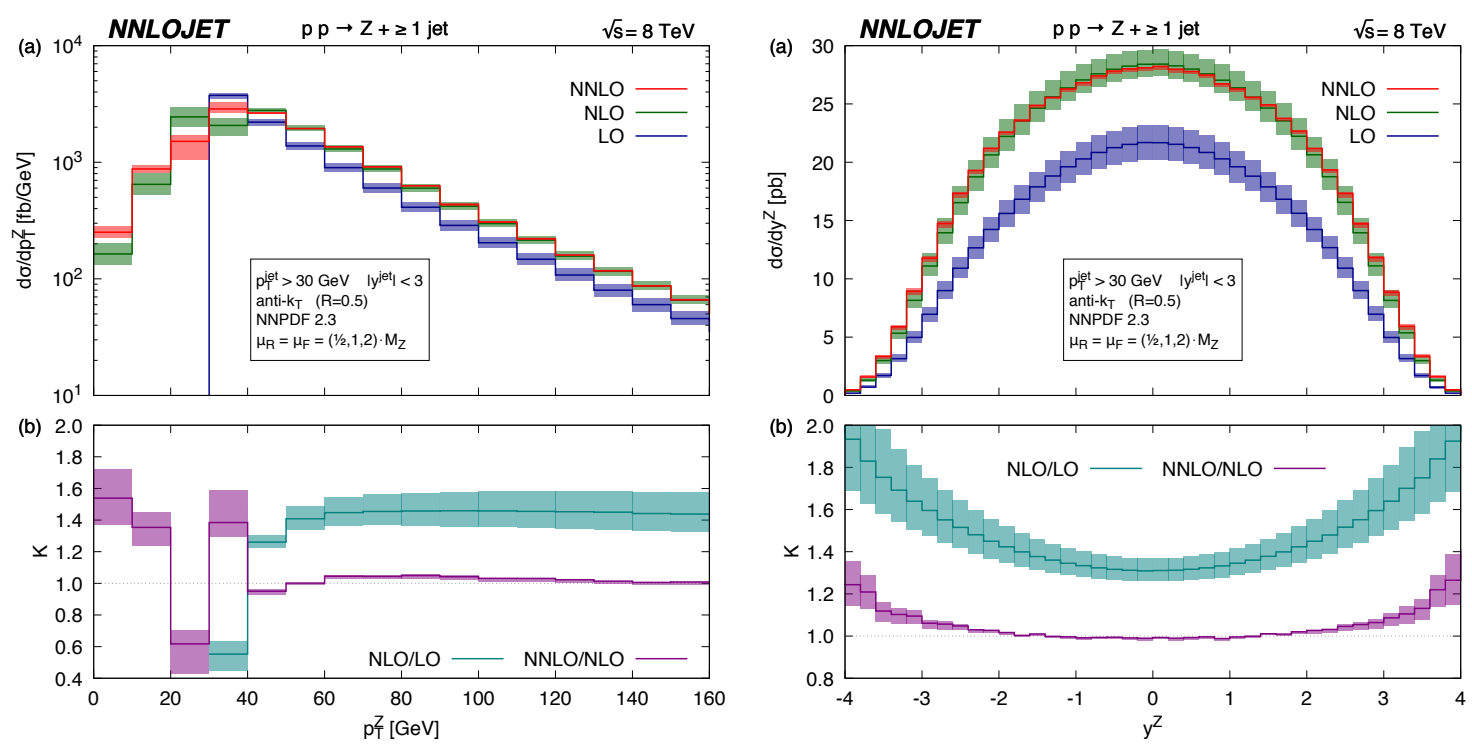

Figure 1: The transverse momentum (left) and rapidity (right) distribution of the $\mathrm{Z}$ boson in inclusive $Z+$ jet production in $p p$ collisions with $\sqrt{s}=8 \mathrm{TeV}$. The absolute distributions at LO (blue), NLO (green), and NNLO (red) are shown in the top panels. The bottom frames display the ratios of different perturbative orders: NLO to LO (turquoise) and NNLO to NLO (mauve).

corrections are very small with a reduced scale dependence. We note that the corrections on differential distributions are not always uniform and that a naive rescaling of lower-order predictions with global $K$-factors will not be able to capture these features.

\section{Inclusive Z-boson production at non-vanishing transverse momentum}

The transverse momentum of the di-lepton system in inclusive Z-boson production is caused by partonic recoil and is thus determined by QCD dynamics. One of the main motivations to study this observable, in particular multi-differentially, is its sensitivity to the gluon distribution which is still poorly constrained at high $x$ values. It is important to note that predictions which are accurate to NNLO in QCD for the inclusive production of $\mathrm{Z}$ bosons are only NLO-accurate in this observable due to the implicit requirement of a partonic recoil. ATLAS and CMS both observed a tension between their measurements and existing NLO QCD predictions, highlighting the potential importance of higher order corrections to this process.

Building upon our calculation of the NNLO QCD corrections to Z + jet discussed in the previous section, we exploit our highly flexible NNLOJET numerical code to predict the Z-boson transverse momentum distribution to NNLO accuracy. To this end, we consider a setup that is fully inclusive with respect to QCD radiation and instead impose a small transverse momentum cut on the $\mathrm{Z}$ boson, enforcing the presence of a non-vanishing hadronic recoil.

The experimental measurement of the transverse momentum of the $\mathrm{Z}$ boson, $p_{\mathrm{T}}^{\mathrm{Z}}$, is presented in the form of fiducial cross sections for a restricted kinematical range of the final state leptons. We compare our NNLO calculation to data by considering the same event selection cuts as used in the ATLAS [12] and CMS [13] analyses based on data collected in Run I of the LHC with $\sqrt{s}=8 \mathrm{TeV}$. 


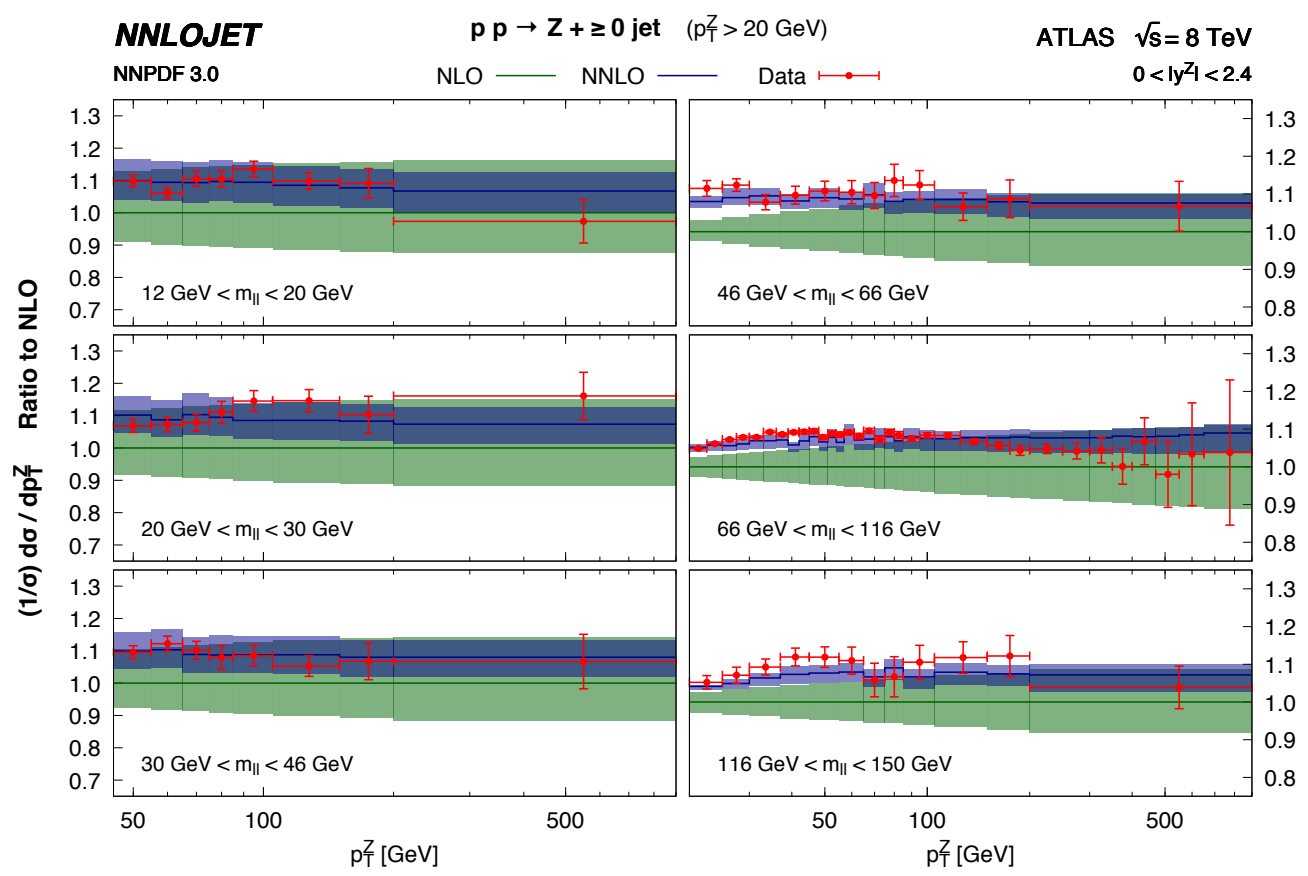

Figure 2: The normalised double-differential transverse momentum distribution for the $\mathrm{Z}$ boson in windows of invariant mass of the leptons, $m_{\ell \ell}$, with a rapidity cut on the $\mathrm{Z}$ boson of $\left|y^{\mathrm{Z}}\right|<2.4$. The ATLAS data is taken from [12]. The green bands denote the NLO prediction with scale uncertainty and the blue bands show the NNLO prediction with scale uncertainty.

In order to reduce the systematic uncertainty on the measurement, in particular the luminosity uncertainty of about $3 \%$, the transverse momentum distribution is commonly normalised to the inclusive production cross section, $(1 / \sigma) \cdot \mathrm{d} \sigma / \mathrm{d} p_{\mathrm{T}}^{\mathrm{Z}}$. We compute the NNLO corrections to the unnormalised $p_{\mathrm{T}}^{\mathrm{Z}}$ distribution using our recent calculation for $\mathrm{Z}+$ jet production by imposing a transverse momentum cut of $20 \mathrm{GeV}$, while the normalisation is obtained from the Drell-Yan cross section. As discussed in Ref. [11], the inclusion of the NNLO QCD effects does not fully resolve the tension with the data for the unnormalised $p_{\mathrm{T}}^{\mathrm{Z}}$ distribution. However, considering normalised distributions leads to a substantial improvement in the agreement between theory and data as will be discussed following. For the respective comparison of unnormalised distributions and further results we refer to Ref. [11], where also more details are given.

In Fig. 2 we present the normalised double-differential distribution with respect to the transverse momentum of the $\mathrm{Z}$ boson and the invariant mass of the lepton pair, $m_{\ell \ell}$, normalised to the NLO prediction and compare it to the ATLAS data [12]. The bands on the theory curves represent the scale uncertainty as obtained through a variation of the renormalisation and factorisation scale by a factor in the range $[1 / 2,2]$ from the central scale choice of $\mu=E_{\mathrm{T}}=\sqrt{m_{\ell \ell}^{2}+\left(p_{\mathrm{T}}^{\mathrm{Z}}\right)^{2}}$ and are greatly reduced by moving to NNLO from NLO. Tension between the NLO prediction and the data is seen in the three higher mass bins where the data is significantly overshooting the theory prediction. The NNLO corrections in these bins are not uniform and have a large positive correction at small $p_{\mathrm{T}}^{\mathrm{Z}}$. This is particularly apparent for the $m_{\ell \ell}$ bin containing the Z-boson resonance. The tension observed at NLO is completely resolved by the inclusion of the NNLO corrections and we 


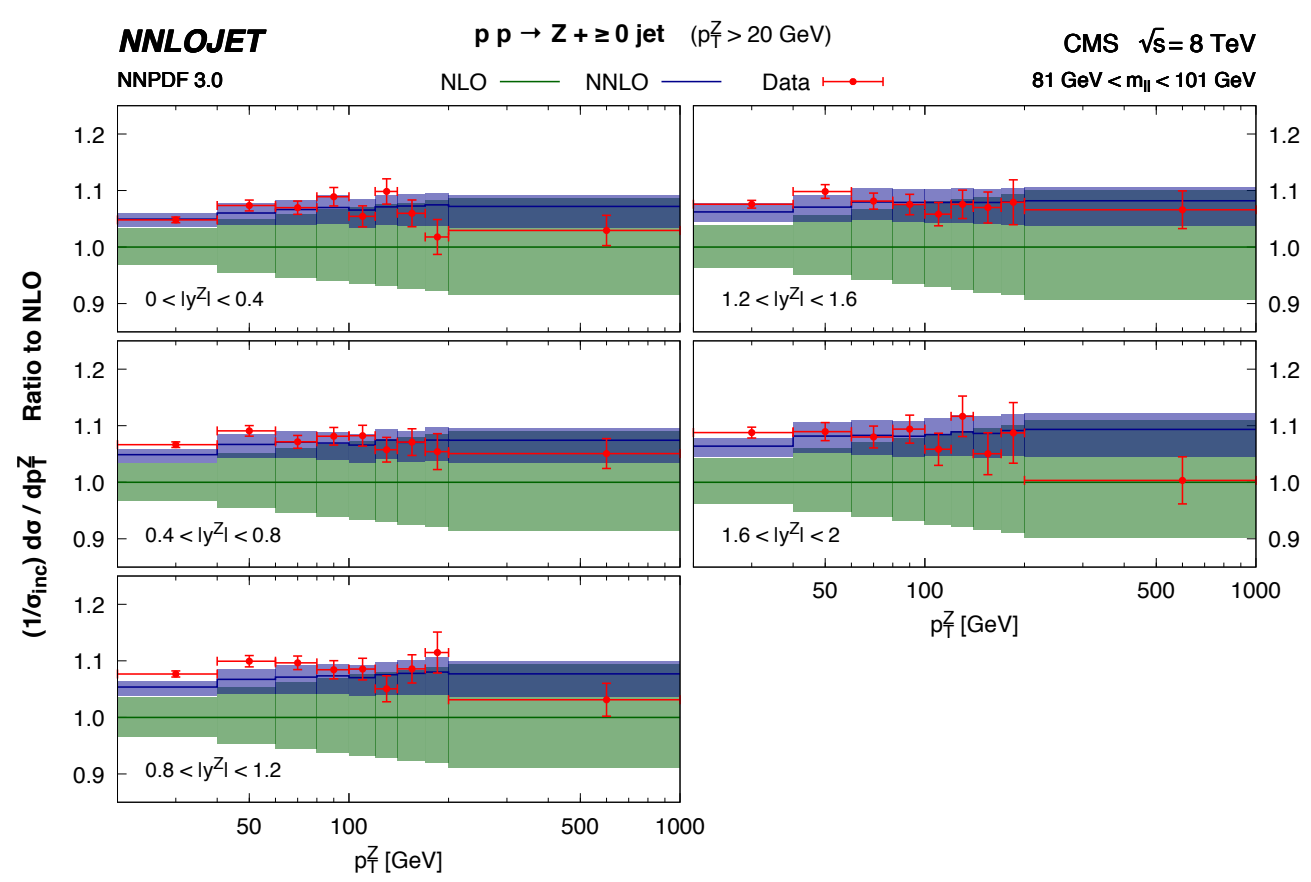

Figure 3: The normalised double-differential transverse momentum distribution for the $\mathrm{Z}$ boson in windows of rapidity of the $\mathrm{Z}$ boson, $y^{\mathrm{Z}}$, with an invariant mass cut on final state leptons of $81 \mathrm{GeV}<m_{\ell \ell}<101 \mathrm{GeV}$. The CMS data is taken from [13]. The green bands denote the NLO prediction with scale uncertainty and the blue bands show the NNLO prediction.

see very good agreement between data and theory.

Figure 3 shows the comparison of the CMS measurement [13] to our calculation for the normalised double-differential distribution with respect to $p_{\mathrm{T}}^{\mathrm{Z}}$ and the rapidiy of the $\mathrm{Z}$ boson $\left(y^{\mathrm{Z}}\right)$, as a ratio to the NLO prediction. The tension between the theoretical prediction and the data is similar to the ATLAS analysis [12], where the measured data points lie systematically above the prediction. The NNLO corrections are relatively uniform in rapidity and transverse momentum in the considered kinematic region and amount to about positive $5-10 \%$ corrections, with a decreased residual theory uncertainty of 2-4\%. Again, the inclusion of the NNLO corrections completely removes the tension with the data and we obtain excellent agreement.

\section{Conclusions}

We have presented the NNLO QCD corrections to the $\mathrm{Z}+$ jet process and to the production of Z-bosons at high transverse momentum. This calculation is performed using the parton-level Monte Carlo generator NNLOJET which implements the antenna subtraction method for NNLO calculations of hadron collider observables. We performed a comparison of the theory prediction to the ATLAS [12] and CMS [13] studies of Z-boson transverse momentum distribution based on the LHC Run I data. Although a residual tension between the NNLO QCD theory prediction and experiment remains for the unnormalised inclusive- $p_{\mathrm{T}}^{\mathrm{Z}}$ distributions, this is considerably alleviated when the distributions are normalised by the inclusive cross section. 


\section{Acknowledgments}

The authors would like to acknowledge the support provided by the GridPP Collaboration. This research was supported in part by the National Science Foundation under Grant NSF PHY1125915, in part by the Swiss National Science Foundation (SNF) under contracts 200020-162487 and CRSII2-160814, in part by the UK Science and Technology Facilities Council, in part by the Research Executive Agency (REA) of the European Union under the Grant Agreement PITN-GA2012-316704 ("HiggsTools") and the ERC Advanced Grant MC@ NNLO (340983).

\section{References}

[1] G. Aad et al. [ATLAS Collaboration], JHEP 1307, 032 (2013) [arXiv:1304.7098 [hep-ex]].

[2] S. Chatrchyan et al. [CMS Collaboration], Phys. Lett. B 722 (2013) 238 [arXiv:1301.1646 [hep-ex]]; Phys. Rev. D 88 (2013) 11, 112009 [arXiv:1310.3082 [hep-ex]]. V. Khachatryan et al. [CMS Collaboration], Phys. Rev. D 91 (2015) 5, 052008 [arXiv:1408.3104 [hep-ex]].

[3] W. T. Giele, E. W. N. Glover and D. A. Kosower, Nucl. Phys. B 403 (1993) 633 [hep-ph/9302225].

[4] A. Denner, S. Dittmaier, T. Kasprzik and A. Mück, JHEP 1106 (2011) 069 [arXiv:1103.0914 [hep-ph]].

[5] S. Kallweit, J. M. Lindert, P. Maierhöfer, S. Pozzorini and M. Schönherr, JHEP 1604 (2016) 021 [arXiv:1511.08692 [hep-ph]].

[6] A. Gehrmann-De Ridder, T. Gehrmann, E. W. N. Glover, A. Huss and T. A. Morgan, Phys. Rev. Lett. 117 (2016) 022001 [arXiv:1507.02850 [hep-ph]].

[7] R. Boughezal, J. M. Campbell, R. K. Ellis, C. Focke, W. T. Giele, X. Liu and F. Petriello, Phys. Rev. Lett. 116 (2016) 152001 [arXiv:1512.01291 [hep-ph]].

[8] A. Gehrmann-De Ridder, T. Gehrmann, E.W.N. Glover, A. Huss and T.A. Morgan, PoS RADCOR2015 (2016) 075 [arXiv:1601.04569 [hep-ph]].

[9] A. Gehrmann-De Ridder, T. Gehrmann and E. W. N. Glover, JHEP 0509 (2005) 056 [hep-ph/0505111]; Phys. Lett. B 612 (2005) 49 [hep-ph/0502110]; Phys. Lett. B 612 (2005) 36 [hep-ph/0501291]; A. Daleo, T. Gehrmann and D. Maitre, JHEP 0704 (2007) 016 [hep-ph/0612257]; A. Daleo, A. Gehrmann-De Ridder, T. Gehrmann and G. Luisoni, JHEP 1001 (2010) 118 [arXiv:0912.0374]; T. Gehrmann and P.F. Monni, JHEP 1112 (2011) 049 [arXiv:1107.4037]; R. Boughezal, A. Gehrmann-De Ridder and M. Ritzmann, JHEP 1102 (2011) 098 [arXiv:1011.6631]; A. Gehrmann-De Ridder, T. Gehrmann and M. Ritzmann, JHEP 1210 (2012) 047 [arXiv:1207.5779]; J. Currie, E.W.N. Glover and S. Wells, JHEP 1304 (2013) 066 [arXiv:1301.4693 [hep-ph]].

[10] S. Catani and B. R. Webber, JHEP 9710 (1997) 005 [hep-ph/9710333].

[11] A. Gehrmann-De Ridder, T. Gehrmann, E. W. N. Glover, A. Huss and T. A. Morgan, JHEP 1607 (2016) 133 [arXiv:1605.04295 [hep-ph]]; A. Gehrmann-De Ridder, T. Gehrmann, E. W. N. Glover, A. Huss and T. A. Morgan, arXiv:1610.01843 [hep-ph].

[12] G. Aad et al. [ATLAS Collaboration], arXiv:1512.02192 [hep-ex].

[13] V. Khachatryan et al. [CMS Collaboration], Phys. Lett. B 749 (2015) 187 [arXiv:1504.03511 [hep-ex]]. 